\title{
Numerical Study of Electrostatic Field Distortion on LPTPC End-Plates based on Bulk Micromegas Modules
}

\author{
Purba Bhattacharya ${ }^{1}$, Deb Sankar Bhattacharya ${ }^{2,4}$, Supratik Mukhopadhyay ${ }^{2, a}$, Nayana Majumdar2, Sudeb \\ Bhattacharya ${ }^{3}$, Paul Colas ${ }^{4}$, and David Attié ${ }^{4}$ \\ ${ }^{1}$ School of Physical Science, National Institute of Science Education and Research, Jatni, Khurda, India 752050 \\ ${ }^{2}$ Applied Nuclear Physics Division, Saha Institute of Nuclear Physics, 1/AF, Bidhannagar, Kolkata, India 700064 \\ ${ }^{3}$ Retired Professor, Applied Nuclear Physics Division, Saha Institute of Nuclear Physics, 1/AF, Bidhannagar, Kolkata, India 700064 \\ ${ }^{4}$ DSM/IRFU, CEA/Saclay, F-91191. Gif-sur-Yvette, CEDEX, France
}

\begin{abstract}
The R\&D activities for the linear collider TPC (LC-TPC) are currently working on the adoption of the micro pattern devices for the gaseous amplification stage. Several beam tests have been carried out at DESY with a $5 \mathrm{GeV}$ electron beam in a $1 \mathrm{~T}$ superconducting magnet. We worked on a large prototype TPC with an end-plate that was built, for the first time, using seven resistive bulk Micromegas modules. During experiments, reduced signal sensitivity was observed at the boundary of these modules. Electrostatic field distortion near the module boundaries was considered to be the possible major reason behind these observations. In the present work, we will explore this hypothesis through numerical simulation. Our aim has been to understand the origin of distortions observed close to the edges of the test beam modules and to explore the possibility of using the Garfield simulation framework for investigating a phenomenon as complex as distortion.
\end{abstract}

\section{Introduction}

A Time Projection Chamber (TPC) [1] is envisaged just beyond the vertex detector of the proposed linear collider [2]. Because of the high flux environment, Micro-Pattern Gaseous Detectors (MPGDs) [3] have been proposed to build the end-plates of the TPC [4]. The end-plates are expected to be made of a number of MPGD modules, as shown in figure 1(a), because of the large area coverage and their geometry [5]. Since 2008, the LC-TPC collaboration has been involved in the design, development and test of a Large Prototype TPC (LP-TPC) with amplification systems based on different MPGDs and corresponding read-out schema.

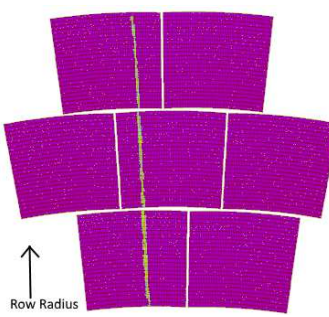

(a)

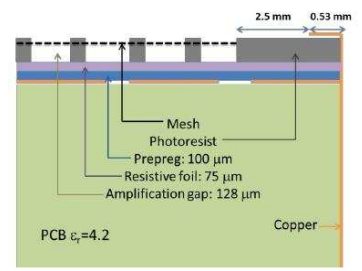

(b)
Figure 1. (a) A reconstructed track on the TPC end-plate made of seven Micromegas modules; (b) schematic diagram of a region close to the edge of one of the module, side view.

\footnotetext{
a e-mail: supratik.mukhopadhyay@saha.ac.in
}

For the 2013 February test beam run, Micromegas modules with resistive coating were chosen [7]. These modules have keystone shape $\left(\sim 22 \times 17 \mathrm{~cm}^{2}\right)$, which is similar to the shape foreseen for the ILD-TPC. The Micromegas in each of the modules has an amplification gap of $128 \mu \mathrm{m}$ and a pitch of $63 \mu \mathrm{m}$. The resistive carbon-loaded Kapton $囚$ layer with a resistivity of about $3 \mathrm{M} \Omega$ /square was chosen as the resistive layer material. Each module had 1726 pads arranged in 24 rows by 72 pads. The pads were all grounded and were read out with the AFTER-based electronics designed for the T2K experiment [6]. The reconstruction of a typical track on theTPC end-plate, consisting of seven modules, is shown in figure 1(a). The close-up schematic diagram of the region near the module edge is shown in figure and 1(b) [7].

The DESY electron beam energy was varied from 1 $5 \mathrm{GeV}$ during the various phases of experiment. The working gaseous mixture in the TPC was $\mathrm{Ar}-\mathrm{CF}_{4}$-Isobutane (95: $3: 2$ ), so-called T2K gas. Data related to cosmic rays, drift velocity estimation, $Z$ scan, phi scan, drift field scan, variation of gain, effect of different magnetic field etc. were recorded and analyzed in detail [7]. During the analysis process, reduced signal sensitivity was observed near the edge of the module [9]. It was also found that the spatial resolutions of all the pads are consistent, except for those near the module edge [7, 8]. Figure 2 shows the distortion (residual) plot versus the row radius without or with magnetic field. The transfer between two detector modules is seen and in-between them, the distortion is larger and leads to ' $\mathrm{S}$ ' shape as shown in figures 2(a) 
( $B=0 \mathrm{~T})$ and figure $2(\mathrm{~b})(B=1 \mathrm{~T})$. It may be noted that the difference between the distortions is expected since in the presence of magnetic field, the transverse diffusion is known to be reduced and the distortion is likely to be influenced by $\vec{E} \times \vec{B}$ effect.

The most likely reason leading to this distortion is the electric field inhomogeneity introduced by the gap between a module and its neighbor. Similar conclusion was arrived at by studying the electric field configuration near the module boundaries with GEM amplification stages in [9] using a very simplified version of the actual experimental setup. In what follows, besides investigating the electric field at the module boundaries for an end-plate having Micromegas modules, we have numerically estimated the residuals for a relatively more realistic geometry and compared our estimates with the experimental data.

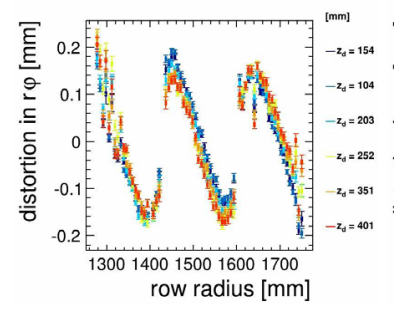

(a)

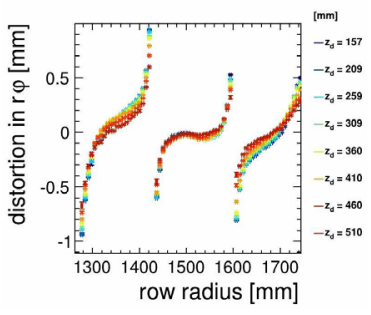

(b)
Figure 2. Distortion (residual) versus row in (a) $B=0 \mathrm{~T}$ and (b) $B=1 \mathrm{~T}$.

\section{Simulation Tools}

The Garfield [10] simulation framework has been used in the following work. Besides neBEM [11], which is used for electrostatic calculations, the Garfield framework provides interfaces to HEED [12] for primary ionization calculation and Magboltz [13] for computing drift, diffusion, Townsend and attachment coefficients.

\section{Simulation Conditions}

The model used for the simulation, although more realistic than the model used in [9], is simpler than that used in the real experimental setup and deals with a much smaller geometry in comparison to the actual system. By comparing the experimental condition, as depicted in figure 1, and the numerical model shown in figure 3 , the following can be pointed out:

- In the place of seven, only three modules have been used.

- Each module is a rectangle of $4.0 \mathrm{~cm} \times 3.7 \mathrm{~cm}$ along $\mathrm{X}-\mathrm{Y}$ plane, in place of keystone shaped modules of $22 \mathrm{~cm} \times 17 \mathrm{~cm}$ matching the r-phi placement of the LP-TPC.

- The actual experiment was performed using a resistive bulk Micromegas, whereas, for this simulation, the standard bulk Micromegas has been considered.
- Instead of cylinders, infinitely thin wire elements have been used to model the micromesh plane.

- A continuous grounded anode plane has been placed $128 \mu \mathrm{m}$ below the mesh plane. A virtual pad structure has been evoked only when necessary.

- Instead of a TPC field cage, a drift plane has been placed $1 \mathrm{~cm}$ above the modules.

- As in the experiment, $3 \mathrm{~mm}$ thick photoresist has been used to support the modules.

- True to the experiment, a copper layer has been connected all around the module to the ground.

- Between the modules there is a gap of $1 \mathrm{~mm}$ which follows the experimental situation.

It should also be mentioned that, for the results presented here, the micromesh plane has been biased with a potential of $-350 \mathrm{~V}$ whereas $-550 \mathrm{~V}$ has been applied to the drift plane. Three different tracks, as shown in figure 3(a), have been considered.

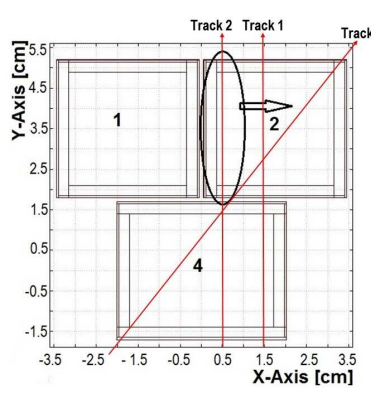

(a)

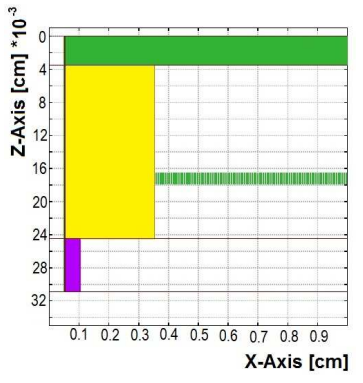

(b)
Figure 3. (a) Top view of the simulated model, (b) closer (marked by the ellipse) side view of the edge of one module. Different tracks used in this simulation are shown in (a).

\section{Results}

\subsection{Electrostatic Field}

Figure 4(a) and figure 4(b) show the field contour along Track 1 and Track 2, respectively. In the vicinity of the gap between the modules, the field is affected by the presence of the additional ground line around the module and the dielectric material. It may be noted from figures 1(b) and $3(\mathrm{~b})$ that the dielectric support is $(2.5+0.55) \mathrm{mm}$, the ground line has $0.55 \mathrm{~mm}$ length in the Y-direction, while the gap between the two modules is $1 \mathrm{~mm}$. So, after the micromesh ends on one module, there is a gap of $4.5 \mathrm{~mm}$ before the micromesh in the next module begins. The gap lies in between $1.7 \mathrm{~cm}$ to $1.8 \mathrm{~cm}$ in the $\mathrm{Y}$ direction. The transverse component of the field, in the region between $1.2 \mathrm{~cm}$ to $2.3 \mathrm{~cm}$ is significantly large. Since Track 2 is close to the module edge in the X-direction as well, the field lines are found to be distorted further. This distortion of the field is very likely to affect the electrons drifting towards the read-out module. Similar estimates for GEMbased TPC end-plates [9] were made earlier, as mentioned 


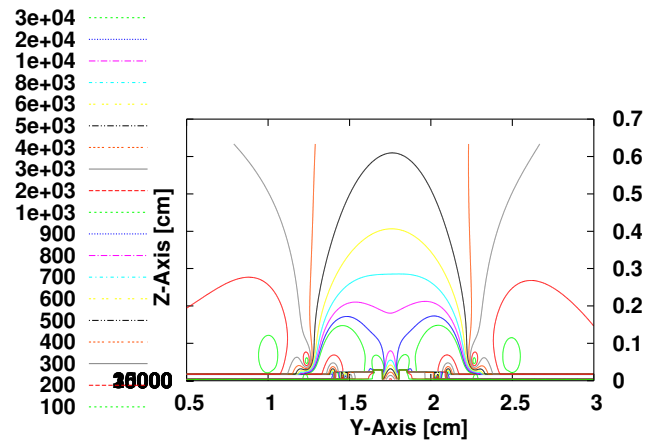

(a)

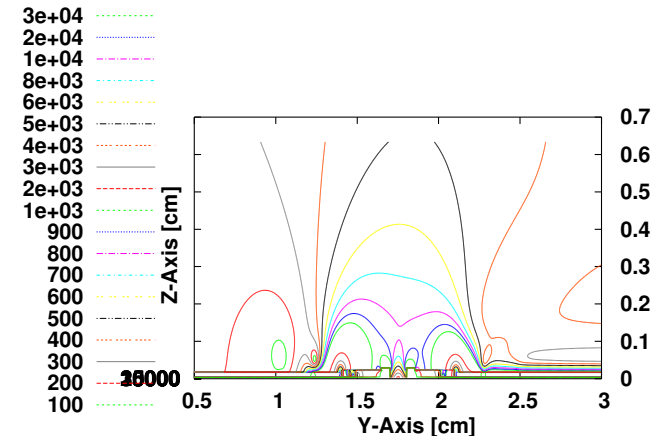

(b)

Figure 4. Field contours on a YZ plane along Tracks (a) 1 and (b) 2.

above. Although overall features of the electrostatic field, electron drift and diffusion are the same, no direct comparison can be made since the geometries of the modules and the approximations involved are of a different nature.

\subsection{Drift Lines}

For the present calculation, $\mathrm{T} 2 \mathrm{~K}$ gas (Argon $95 \%, \mathrm{CF}_{4} 2 \%$, Isobutane $2 \%$ ) has been considered. To study the effects of the field, electron tracks along Track 1 at a distance of $5 \mathrm{~mm}$ above the module have been considered. The electrons from these predetermined tracks are allowed to drift towards the read-out plane (figure 5(a)). At the edge of the module, the drift lines get distorted. It is observed from figure 5 that a significant number of electrons are lost on the additional ground and the dielectric material. From the drift lines, it is clear that the number of electrons at the read-out pads close to the module edge is less in comparison to that at the central part of the module. The presence of the magnetic field $(1 \mathrm{~T})$ reduces the diffusion as shown in figure 5(b), as expected. From a quantitative estimate of the efficiency carried out using a virtual $4 \mathrm{~mm}$ long pad structure on the anode plane, it has been found that without magnetic field, the pad adjacent to the module edge has little charge collection, while the second pad has close to $60 \%$ collection. With magnetic field, the pad adjacent to the edge has a charge collection efficiency of around $40 \%$, while the second pad is found to work almost at the full efficiency.

\subsection{Calculation of Residue}

In experiments, the residue is calculated to get an idea of the deviation of the measured hit position in the readout pad with respect to the position of the real track. Similarly, we have estimated residues for Track 3 for all the electrons that reach the anode plane by estimating the difference between the start and end points of these electrons. Since no pad structure has been considered, and the resistive nature of the anode plane has been ignored, the term intrinsic residue has been used to mean the numerical estimate. The patterns that emerge from these estimates follow the same trend as obtained through the analysis of real data.

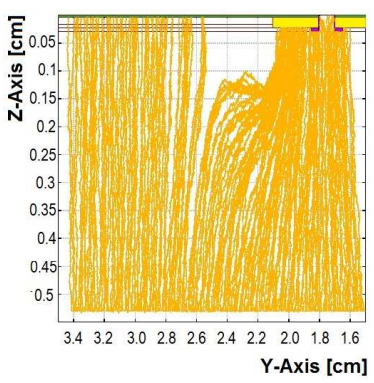

(a)

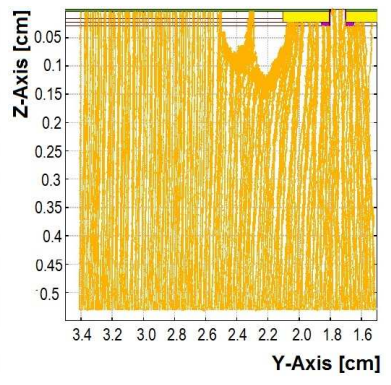

(b)
Figure 5. Electron drift lines along Track 1 in (a) $B=0 \mathrm{~T}$, (b) $B=1 \mathrm{~T}$.

From the residue histograms (figures 4.3, 4.3), it can be observed that residues reduce significantly due to the presence of a magnetic field. This is easily explained by noting that magnetic field decreases diffusion since it acts as an additional constraint that makes the charged particle follow electric field lines. The X-residual plots for $B=0 \mathrm{~T}$ (figure 4.3 and $B=1 \mathrm{~T}$ (figure 4.3 ) reflects this fact once again. The effect of magnetic field is striking and the sign of the residues are found to change with and without magnetic field. Interestingly, this change of sign is also observed in the experimental data. It may be noted here that, though our simulation follows the experimental trend, the value of the distortion is larger in the numerical estimation, the maximum in residue being close to $1 \mathrm{~mm}$ instead of around $0.5 \mathrm{~mm}$ as found in the experimental data.

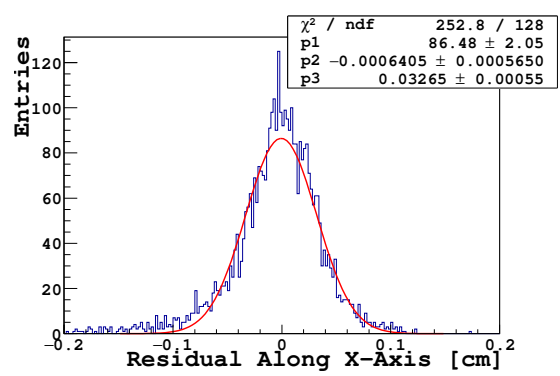

Figure 6. Histogram of residue alongX-Axis in $B=0 \mathrm{~T}$. 


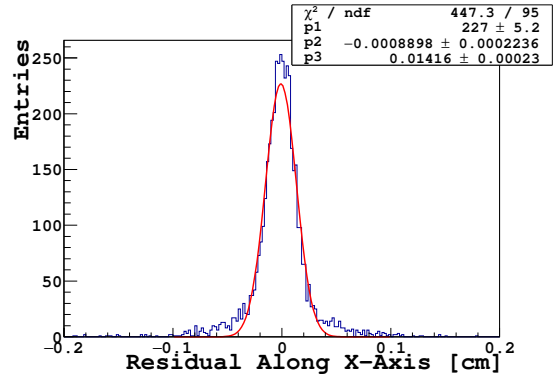

Figure 7. Histogram of residue along X-Axis in $B=1 \mathrm{~T}$.

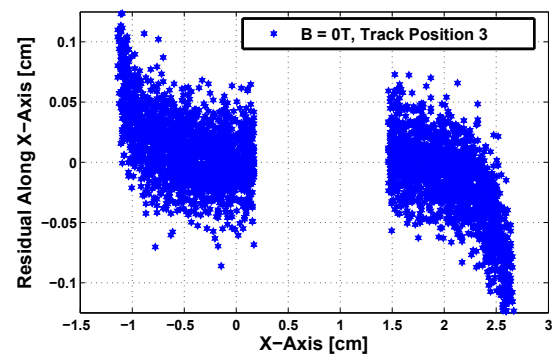

Figure 8. Residual along $\mathrm{X}$-Axis in $B=0 \mathrm{~T}$ for Track 3 .

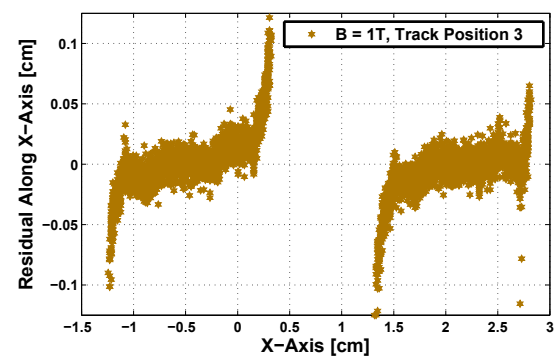

Figure 9. Residual along $\mathrm{X}$-Axis in $B=1 \mathrm{~T}$ for Track 3 .

The differences between the two estimates probably arise from the simplifications adopted in the numerical model. Besides the smallness of the modules, lack of resistive anode and the thin-wire assumptions are probably the most significant ones. Among these, the resistive anode in a real experiment is expected to improve reconstruction of the hit point and, thus, reduce the residue in the experimental data. The thin-wire assumption is likely to affect the transmission of electrons through the mesh. However, despite the short-comings arising out of these simplifying assumptions, this preliminary study is capable of bringing out the main features of the experimental observation.

\section{Conclusion}

Following the experimental activities and related data analysis, we have investigated the origin of the so-called distortions observed close to the module edges. We have been able to numerically simulate the observed patterns quite successfully, although we have not made any attempt to achieve quantitative agreement with the experimental data. Our computations indicate that the inhomogeneity of the electric field close to the module edges leads to a loss of efficiency of few pads close to the edge and also leads to the distortion in residue observed in the experimental data. The presence of magnetic field complicates the matter through the $E \times B$ effect, since the two fields are no more parallel (due to the fact that the $E$-field is distorted). The obtained qualitative agreements encourage us to continue with the study and, if possible, propose module design modifications that can alleviate the problem. At least, we should be able to suggest corrections that can be applied during the data analysis phase to take care of this issue. In future, the simulations will be made more realistic by making the end-plate geometry closer to the real one and by making the anode resistive. In addition, possible suitable design modifications leading to less inhomogeneity at the module edges will be investigated.

\section{Acknowledgements}

This work has partly been performed in the framework of the RD51 Collaboration. We wish to acknowledge the members of the RD51 Collaboration for their help and suggestions. We thank our collaborators from the ILC-TPC collaboration for their help and suggestions. We gratefully acknowledge IFCPAR/CEFIPRA (Project No. 4304-1) for partial financial support. Finally, we thank our respective Institutions for providing us with the necessary facilities.

\section{References}

[1] D. R. Nygren et al., Physics Today 3146 (1978).

[2] http://www.lctpc.org/

[3] M. Hoch, Nucl. Instrum. Method A 5351 (2004).

[4] P. Schade et al., Nucl. Instrum. Meth. A 628128 (2011).

[5] D. Attié, Jour. Instrum. 6 C01007 (2011).

[6] P. Baron et al., IEEE Trans. Nucl. Sci. 551744 (2008).

[7] W. Wang, Ph. D Thesis, Univ. of Paris-Sud, (2013).

[8] D.S.Bhattacharya, Proc. Science (EPS-HEP2015) 277

[9] T. Behnke et al., arXiv:1309.1370v2 (2013).

[10] http://cern.ch/garfield.

[11] http://cern.ch/neBEM.

[12] http://cern.ch/heed.

[13] http://cern.ch/magboltz. 